\title{
Pericardial tamponade due to a ruptured mediastinal hydatid cyst
}

\author{
Weimin Zhang ${ }^{1}$, Feng-xia Wang ${ }^{2}$, Ming-ming Zhang ${ }^{3}$, Renati Imam $^{3}$, Tao Zhu ${ }^{3}$, Hao Wen \\ ${ }^{4}$, and Qiang Huo ${ }^{5}$ \\ ${ }^{1}$ The First Affiliated Hospital of Xinjiang Medical University, Urumqi, Xinjiang 830011, \\ P.R. China \\ ${ }^{2}$ Department of cardiology, People's Hospital of Xinjiang Uygur Autonomous Region, \\ Urumqi, Xinjiang 830001, P.R. China \\ ${ }^{3}$ Department of Cardiac Surgery, The First Affiliated Hospital of Xinjiang Medical \\ University, Urumqi, Xinjiang 830011, P.R. China \\ ${ }^{4}$ Xinjiang Key Lab of Fundamental Medical Research and Xinjiang Hydatid Clinical \\ Research Institute, The First Affiliated Hospital of Xinjiang Medical University, Urumqi, \\ Xinjiang 830001, P.R. China. \\ ${ }^{5}$ The First Affiliated Hospital of Xinjiang Medical Universit
}

November 18, 2020

\begin{abstract}
Hydatid disease is a zoonosis caused by Echinococcus granulosus. It is seen most frequently in the liver and lungs in adults. The mediastinal location of this disease is rare. Herein, we report the case of a 38-year-old male with signs of heart failure related to a rupture of a mediastinal hydatid cyst with pericardial tamponade. The diagnosis was confirmed by echocardiography, computed tomography, and hydatic serology, and the patient was operated and put on albendazole for 3 months with favorable clinical course.
\end{abstract}

\section{Hosted file}

pericardial tamponade due to ruptured mediastinal hydatid cyst - grammarly - clean.pdf available at https://authorea.com/users/376831/articles/493689-pericardial-tamponade-due-toa-ruptured-mediastinal-hydatid-cyst 


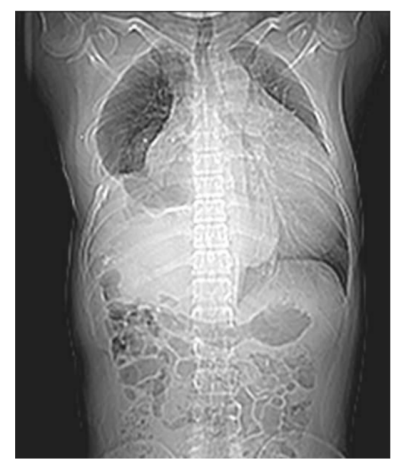

Wei-Min Zhang etal,. Figure1

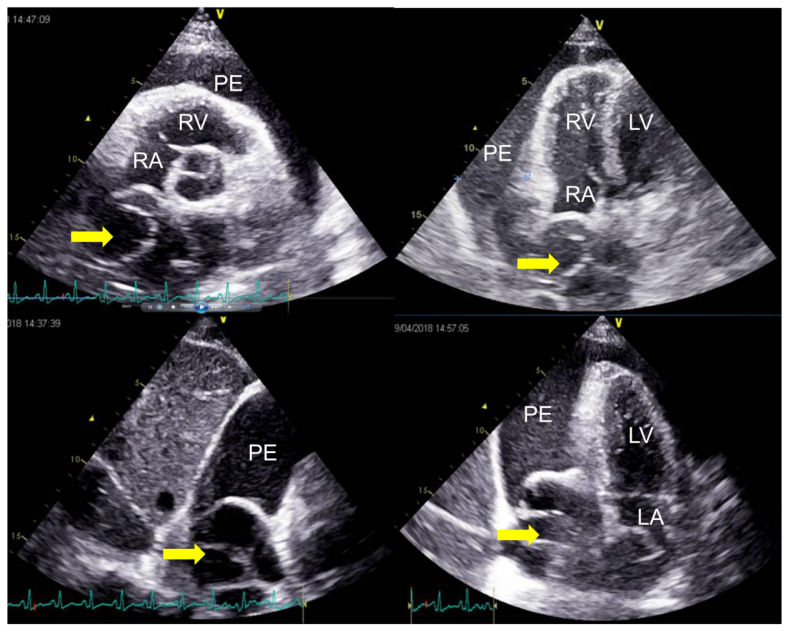

Two-dimensional TTE image: yellow short axis view showing hydatid cyst; $L A=$ left atrium; $R A=$ right atrium; $L V=$ left ventricle; $R V=$ right ventricle; $P E=$ pericardial effusion

Wei-Min Zhang etal,. Figure2 


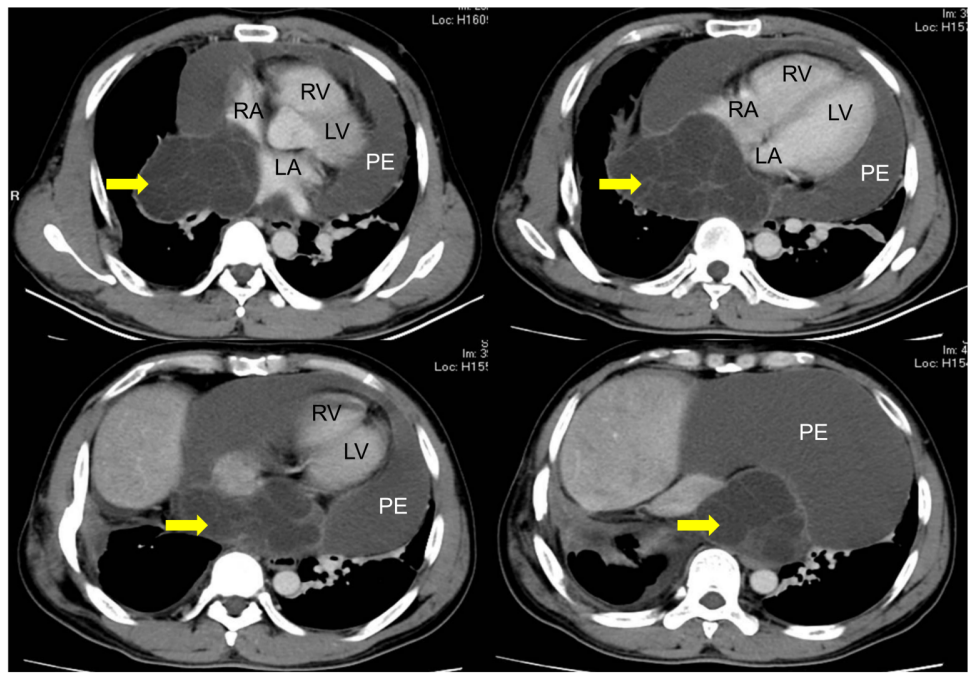

Multislice cardiac computed tomography image : yellow short axis view showing hydatid cyst; $L A=$ left atrium; $R A=$ right atrium; $L V=$ left ventricle; $R V$

$=$ right ventricle; $\mathrm{PE}=$ pericardial effusion

Wei-Min Zhang etal,. Figure3 